\title{
Chronic thromboembolic pulmonary hypertension
}

\author{
Nick H. Kim ${ }^{1}$, Marion Delcroix $\mathbb{1}^{2}$, Xavier Jais ${ }^{3}$, Michael M. Madani ${ }^{4}$, \\ Hiromi Matsubara ${ }^{5}$, Eckhard Mayer ${ }^{6}$, Takeshi Ogo $^{7}$, Victor F. Tapson ${ }^{8}$, \\ Hossein-Ardeschir Ghofrani $\mathbb{1}^{6,9,10,12}$ and David P. Jenkins ${ }^{11,12}$
}

Number 11 in the series

"Proceedings of the 6th World Symposium on Pulmonary Hypertension"

Edited by N. Galiè, V.V. McLaughlin, L.J. Rubin and G. Simonneau

\begin{abstract}
Affiliations: ${ }^{1}$ Dept of Medicine, University of California San Diego, La Jolla, CA, USA. ${ }^{2}$ Dept of Respiratory Diseases, University Hospitals of Leuven and Respiratory Division, Dept CHROMETA, KU Leuven - University of Leuven, Leuven, Belgium. ${ }^{3}$ Université Paris-Sud, AP-HP, Centre de Référence de l'Hypertension Pulmonaire, Service de Pneumologie, Département Hospitalo-Universitaire (DHU) Thorax Innovation (TORINO), Hôpital Bicêtre, Le Kremlin-Bicêtre, France. ${ }^{4}$ Cardiovascular and Thoracic Surgery, University of California San Diego, La Jolla, CA, USA. ${ }^{5}$ National Hospital Organization Okayama Medical Center, Okayama, Japan. ${ }^{6}$ Kerckhoff Clinic Bad Nauheim, University of Giessen, Bad Nauheim, Germany. ${ }^{7}$ Division of Advanced Medical Research in Pulmonary Hypertension, Dept of Pulmonary Circulation, National Cerebral and Cardiovascular Center, Osaka, Japan. ${ }^{8}$ Dept of Medicine, Cedars-Sinai Medical Center, Los Angeles, CA, USA. ${ }^{9}$ University of Giessen and Marburg Lung Centre (UGMLC), Justus-Liebig University Giessen and Member of the German Center for Lung Research (DZL), Giessen, Germany. ${ }^{10}$ Dept of Medicine, Imperial College London, London, UK. ${ }^{11}$ Royal Papworth Hospital, Cambridge, UK. ${ }^{12}$ These two authors contributed equally to this work.
\end{abstract}

Correspondence: Nick H. Kim, Dept of Medicine, University of California San Diego, 9300 Campus Point Drive, MC 7381, La Jolla, CA 92037, USA. E-mail: h33kimaucsd.edu

@ERSpublications

State of the art and research perspectives in chronic thromboembolic pulmonary hypertension, including treatment algorithm http://ow.ly/C3Iy30mfUly

Cite this article as: Kim NH, Delcroix M, Jais X, et al. Chronic thromboembolic pulmonary hypertension. Eur Respir J 2019; 53: 1801915 [https://doi.org/10.1183/13993003.01915-2018].

ABSTRACT Chronic thromboembolic pulmonary hypertension (CTEPH) is a complication of pulmonary embolism and a major cause of chronic $\mathrm{PH}$ leading to right heart failure and death. Lung ventilation/perfusion scintigraphy is the screening test of choice; a normal scan rules out CTEPH. In the case of an abnormal perfusion scan, a high-quality pulmonary angiogram is necessary to confirm and define the pulmonary vascular involvement and prior to making a treatment decision. $\mathrm{PH}$ is confirmed with right heart catheterisation, which is also necessary for treatment determination. In addition to chronic anticoagulation therapy, each patient with CTEPH should receive treatment assessment starting with evaluation for pulmonary endarterectomy, which is the guideline recommended treatment. For technically inoperable cases, $\mathrm{PH}$-targeted medical therapy is recommended (currently riociguat based on the CHEST studies), and balloon pulmonary angioplasty should be considered at a centre experienced with this challenging but potentially effective and complementary intervention. 


\section{Introduction}

Since the 5th World Symposium on Pulmonary Hypertension (WSPH) in 2013, major progress has occurred in the understanding and management of chronic thromboembolic pulmonary hypertension (CTEPH). First, the link between CTEPH and acute pulmonary embolism, and some of the challenges associated with making the connection, will be reviewed. Key diagnostic steps in establishing early and accurate diagnosis will be emphasised. Each component of the current CTEPH treatment approach will be overviewed. Finally, an updated treatment algorithm is proposed taking into account the advances since 2013.

\section{CTEPH and pulmonary embolism}

CTEPH is classified within group $4 \mathrm{PH}$ [1], and is characterised pathologically by organised thromboembolic material and by altered vascular remodelling initiated or potentiated by a combination of defective angiogenesis, impaired fibrinolysis and endothelial dysfunction [2-4]. These changes lead to $\mathrm{PH}$ and ultimately right ventricular failure $[5,6]$. The precise pathogenesis of CTEPH remains unclear, but appears to be incited by acute pulmonary embolism [7].

However, classic risk factors for venous thromboembolism do not appear to increase the risk of CTEPH [8] and there are clear geographic differences in CTEPH epidemiology. An international CTEPH registry (Europe and Canada) indicated that $75 \%$ of patients with CTEPH had a documented antecedent history of acute pulmonary embolism [9], while in Japan, the rates of acute pulmonary embolism preceding CTEPH range from only $15 \%$ to $33 \%[10,11]$. There is an $80 \%$ female preponderance of CTEPH in Japan; these statistics differ significantly from the USA and Europe [9]. A number of abnormal autoimmune, inflammatory and thrombophilia markers have been found in CTEPH patients [2]; it is feasible that variability in this underlying pathological milieu contributes to the variability in the worldwide CTEPH epidemiology. Furthermore, variable gene expression has been demonstrated in pulmonary artery endothelial cells from patients with CTEPH compared with normal controls [12].

In published prospective studies with the diagnosis confirmed by right heart catheterisation (RHC) the incidence of CTEPH after symptomatic acute pulmonary embolism is reported to range from $0.4 \%$ to $6.2 \%$ [13-25], giving a pooled incidence of 3.4\% (95\% CI 2.1-4.4\%) [7]. Since that analysis, a new report from Switzerland screened 508 patients after acute pulmonary embolism over 2 years and found a cumulative incidence of CTEPH confirmed with RHC of just $0.79 \%$ [26].

Determining the precise CTEPH incidence is complex. CTEPH is likely both underdiagnosed and the incidence of CTEPH after acute pulmonary embolism prone to overestimation, making the actual incidence difficult to quantify. Non-specific symptoms, variable rates of antecedent acute pulmonary embolism and the expertise required to read computed tomography pulmonary angiography (CTPA) contribute to underdiagnosis $[27,28]$. Underdiagnosis is further compounded by the infrequent use of lung ventilation/perfusion scintigraphy (V/Q scan) despite guideline recommendations [29, 30]. Approximately 30000 acute pulmonary embolism cases are diagnosed annually in France, with the CTEPH incidence estimated at 3.4\% [31]. GUÉRIN et al. [22] suggested a CTEPH incidence of $4.8 \%$. Neither of these estimates is consistent with the current frequency of newly diagnosed CTEPH. A limitation of the numerous CTEPH incidence reports after acute pulmonary embolism may be attributed to an unrecognised amalgam of incident and prevalent cases [22].

In terms of reducing the risk of CTEPH following acute pulmonary embolism, no prospective randomised acute pulmonary embolism trials have examined systemic or catheter-based thrombolysis or clot extraction with RHC as an outcome measure in patients with persistent symptoms. Claims have been made that the incidence of CTEPH in patients receiving thrombolytic therapy is reduced, but end-points such as an echocardiogram-derived systolic pulmonary arterial pressure (sPAP) of $40 \mathrm{mmHg}$ do not define $\mathrm{PH}$ or CTEPH [32]. Systemic thrombolysis failed to reduce the risk of CTEPH in intermediate/high-risk (submassive) pulmonary embolism patients in the 3-year follow-up of the PEITHO trial (average sPAP at follow-up was around $31 \mathrm{mmHg}$ in each group) [33]. To date, there is no proof that aggressive treatment of acute pulmonary embolism can prevent CTEPH.

\section{Chronic thromboembolic disease}

Chronic thromboembolic disease (CTED) is characterised by similar symptoms and perfusion defects, but without $\mathrm{PH}$ at rest. Currently a new threshold for PH (mean PAP (mPAP) $>20 \mathrm{mmHg}$ ) and pre-capillary $\mathrm{PH}$ (combination of mPAP $>20 \mathrm{mmHg}$, pulmonary arterial wedge pressure $\leqslant 15 \mathrm{mmHg}$ and pulmonary vascular resistance (PVR) $\geqslant 3$ Wood Units) has been proposed by the 6th WSPH Task Force on $\mathrm{PH}$ diagnosis and classification [1]. While there is good evidence to suggest these new thresholds, the consequences for CTEPH and CTED, respectively, are not yet established. In the future, however, these new thresholds might also be applied to group $4 \mathrm{PH}$. Exercise limitation in CTED has been attributed either to exercise-induced $\mathrm{PH}$, with an increased slope of the pulmonary arterial pressure-flow relationship, or to 
dead-space ventilation, with increased ventilatory equivalents for carbon dioxide $[34,35]$. Both new and worsened dyspnoea and persistent perfusion defects are often encountered after acute pulmonary embolism, in, respectively, $30 \%$ and $30-50 \%$ of patients, which makes the recognition of CTED challenging [18, 36, 37]. Cardiopulmonary exercise tests and echocardiographic evaluations are recommended to exclude patients in whom symptoms are related to lung disease, left heart disease, obesity or deconditioning. A tentative, comprehensive definition of CTED is proposed in table 1. Selected patients with CTED may benefit from pulmonary endarterectomy (PEA) as shown in a series of 42 patients out of 1019 who underwent surgery in the UK reference centre [38]. Post-operative improvement in symptoms, functional class and quality of life were reported. However, while there was no in-hospital mortality, major complications occurred in $40 \%$ of the cohort (small subdural haematomas, tracheostomy). Although endarterectomy is aimed to prevent subsequent disease, the natural history of CTED is unknown and there is no evidence that CTED evolves to CTEPH. At present, patients with CTED represent a group in need of both symptom relief and better understanding of their disorder. CTEPH treatment guidelines should not be applied to CTED.

\section{Diagnosis of CTEPH}

A normal V/Q scan effectively excludes CTEPH with a sensitivity of $90-100 \%$ and a specificity of $94-$ $100 \%[39,40]$. In a study of confirmed cases of CTEPH, V/Q scan was found to be superior to CTPA with a sensitivity of $97.4 \%$ versus $51 \%$ [39]. This difference has narrowed as CT technology and interpretation have advanced. Indeed, a more recent study has shown that both V/Q scan and CTPA are accurate methods for the detection of CTEPH with excellent diagnostic efficacy (100\% sensitivity, 93.7\% specificity and $96.5 \%$ accuracy for $V / Q$ scan; $96.1 \%$ sensitivity, 95.2\% specificity and 95.6\% accuracy for CTPA) [40]. However encouraging, V/Q scan remains the preferred initial imaging test for CTEPH screening $[5,29]$. Recent retrospective studies have also assessed the diagnostic accuracy of three-dimensional dynamic contrast-enhanced lung perfusion magnetic resonance imaging (MRI) against planar V/Q scan or SPECT (single photon emission CT) scan as a screening tool for CTEPH [41, 42]. These studies demonstrated that dynamic contrast-enhanced lung perfusion MRI has a similar sensitivity (97\%) for diagnosing CTEPH when compared with planar VIQ scan and a higher sensitivity (100\% versus $97 \%$ ) when compared with SPECT scan. Prospective studies examining the value of lung perfusion MRI and SPECT scan as a screening test for CTEPH are required to address the clinical utility (including their costs) and diagnostic performance of these modalities.

Digital subtraction angiography (DSA) had been considered the gold standard for characterising vessel morphology in CTEPH, but is being challenged by advances in non-invasive modalities. CTPA is currently widely used for assessment of operability. CTPA in more recent reports has a high sensitivity and specificity in detecting chronic thromboembolic lesions at the main/lobar (89-100\% and 95-100\%, respectively) and segmental ( $84-100 \%$ and $92-99 \%$, respectively) levels [43-45]. CTPA can also be valuable by revealing bronchial artery collaterals, which can correlate with more central disease [46], and by evaluating the lung parenchyma and mediastinum. Advanced CT technologies, including dual-energy CT (DECT), ECG-gated area detector CT (ADCT), cone-beam CT (CBCT) and contrast-enhanced magnetic resonance pulmonary angiography, are emerging as valuable modalities for detailing the pulmonary vasculature. With advances with distal endarterectomy and the advent of balloon pulmonary angioplasty (BPA), and general focus on more distal vascular assessment, conventional DSA may not always be suitable for providing fine details. More selective segmental angiography, CBCT and ADCT may be better for pre-BPA planning by providing greater resolution than conventional DSA, particularly in the more distal vessels [47]. These imaging techniques are not widely available and require expertise.

TABLE 1 Chronic thromboembolic disease (CTED) compared with chronic thromboembolic pulmonary hypertension (CTEPH)

Diagnostic criteria

Symptoms
PH
RHC at exercise
V/Q scan
Angiography (CTPA or DSA)
CPET
TTE

Anticoagulation

CTEPH

CTED

Exercise dyspnoea

Present at rest

Any mismatched perfusion defect Typical findings of CTEPH

At least 3 months
Exercise dyspnoea

Absent at rest

$\mathrm{mPAP} / \mathrm{CO}$ slope $>3 \mathrm{mmHg} \cdot \mathrm{L}^{-1} \cdot \mathrm{min}^{-1}$

Any mismatched perfusion defect

Typical findings of CTEPH

Excluding ventilatory limitation, deconditioning

Excluding left ventricular myocardial or valvular disease At least 3 months

RHC: right heart catheterisation; V/Q: ventilation/perfusion; CTPA: computed tomography pulmonary angiogram; DSA: digital subtraction angiogram; CPET: cardiopulmonary exercise test; TTE: transthoracic echocardiogram; mPAP: mean pulmonary arterial pressure; CO: cardiac output. 


\section{Pulmonary endarterectomy}

PEA should be offered to all eligible patients with CTEPH. The international registry of incident cases of CTEPH reported 3-year survival of $90 \%$ in those operated and $70 \%$ in those not having surgery [48]. Long-term follow-up of a large cohort reported 10-year survival of $72 \%$ (average age 58 years) [49]. Death was attributed to unrelated causes in $49 \%$ of patients; residual PH with PVR $\geqslant 425$ dyn $\cdot \mathrm{s} \cdot \mathrm{cm}^{-5}$ correlated with worse survival [49]. Strict objective definitions of operability remain elusive, but certain features are more likely to predict a good surgical outcome (table 2 ). While select patients may be technically operable, they may not benefit from endarterectomy due to significant comorbidities; the best treatment for such cases remains uncertain. The traditional routine of inserting an inferior vena cava filter (IVC) device prior to endarterectomy has not been formally studied and has been abandoned at the leading surgical centres. In the international registry, IVC filter prior to surgery did not influence long-term survival [48].

The most important surgical advance has been in redefining the distal limits of endarterectomy [50,51]. In expert centres, surgery can be performed successfully in patients with distal chronic thromboembolism [51]. The advances in diagnostics and growing surgical experience have contributed to this success. As a result, the previously published intra-operative classification [52] has been refined to better reflect the current surgical approach and level of revascularisation (table 3) [50]. This also means not all surgical centres will view operability in the same manner [53]. A three-step stratified definition of expert surgical centre has been proposed which factors the following important goals: surgical mortality $(<5 \%)$, surgical volume (more than 50 PEAs per year) and ability to perform segmental endarterectomy [53]. Furthermore, in this era of a comprehensive approach to CTEPH, an expert centre should be capable of evaluating and offering any/all established treatment modalities according to individual need.

The place of PH-targeted medical therapy and BPA relative to surgery is dependent on the anatomical distribution of disease and is not fully defined. Combining endarterectomy with BPA either as a hybrid or stepwise approach is being evaluated at select expert programmes [54]. In the CHEST-1 study, riociguat was beneficial for patients with residual PH after endarterectomy [55]. A trial is needed to clarify if PH-targeted medical therapy prior to endarterectomy in operable patients confers harm or benefit (ClinicalTrials.gov identifier NCT0327357).

\section{Balloon pulmonary angioplasty}

BPA has evolved into an important component of the CTEPH treatment algorithm since the 2012 reports from Japan [56-58]. BPA has been reported to improve haemodynamics, symptoms, exercise capacity and right ventricular function, with significantly lower rates of major complications than compared with the report from 2001 [59-62]. In retrospective analyses, the benefits of BPA also appear to be maintained in the medium term [10,63]. Subsequent publications from Europe report similar results [64-66]. The recent BPA series from Germany is unique as these centres started BPA alongside a well-established PEA programme [67]. Although their complication rates were similar to those from Japan, the magnitude of efficacy (e.g. PVR reduction) was less in comparison with the reports from Japan. The potential explanations offered included the possibility of differences in operability threshold and the variability in the types of patients treated with BPA between centres.

Although these results of BPA are encouraging, the reports are from expert centres and may not be generalisable. Even with the technical refinements, there remains a steep learning curve in order to safely, effectively and consistently perform BPA [68]. A successful BPA requires extensive training and case experience. BPA should be reserved for expert centres, where it should be considered for symptomatic

\section{TABLE 2 Favourable risk-benefit assessment for pulmonary endarterectomy}

Lower risk with predictable good long-term outcome
Characteristics

Higher risk with less predictable long-term outcome (not contraindications)

History
Examination
Comorbidity
Functional limitation
Imaging
Type of disease
Haemodynamics

DVT: deep vein thrombosis; PE: pulmonary embolism; PVR: pulmonary vascular resistance; PA: pulmonary artery.
No history of DVT/PE

No signs of right heart failure None

Functional class II or III Clear disease concordant on all images Bilateral lower lobe disease

PVR $<1000$ dyn $\cdot \mathrm{s} \cdot \mathrm{cm}^{-5}$, in proportion to site and number of obstructions on imaging; higher PA pulse pressure

\section{Signs of right heart failure \\ Significant concomitant lung or left heart disease} Functional class IV

Inconsistency on imaging modalities No disease appreciable in lower lobes PVR $>1200$ dyn $\cdot \mathrm{s} \cdot \mathrm{cm}^{-5}$, out of proportion to site and number of obstructions on imaging; higher PA diastolic pressure 


$\begin{aligned} & \text { TABLE } 3 \text { University of California San Diego chronic thromboembolism (CTE) surgical } \\ & \text { classification }\end{aligned}$
$\begin{array}{ll}\text { Surgical levels } & \text { Location of CTE } \\ \text { Level } 0 & \text { No evidence of thromboembolic disease in either lung } \\ \text { Level I } & \text { CTE starting in the main pulmonary arteries } \\ \text { (Level IC) } & \text { (Complete occlusion of one main pulmonary artery with CTE) } \\ \text { Level II } & \text { CTE starting at the level of lobar arteries or in the main descending pulmonary arteries } \\ \text { Level III } & \text { CTE starting at the level of the segmental arteries } \\ \text { Level IV } & \text { CTE starting at the level of the subsegmental arteries }\end{array}$

Information from [50].

CTEPH patients ineligible for PEA due to distal chronic thromboembolism or persistent/recurrent $\mathrm{PH}$ after surgery. The role of BPA for those with technically operable disease, but who are unsuitable for surgery due to subjective determination or patient refusal, has not been established.

BPA patient selection at an expert centre starts with a multidisciplinary review of all available and pertinent data. Anatomical and functional assessment of pulmonary arteries and lung perfusion are critical to identify the target vessels [69]. A selective pulmonary angiogram of the target vessels will show more details and serves as confirmation prior to intervention during BPA. A selective angiogram may not capture all the distal lesions potentially amenable to BPA, necessitating multiple complementary imaging modalities such as intravascular imaging and pressure gradient analysis to aid in lesion assessment and balloon sizing [70].

BPA complications should be defined and uniformly reported. Unlike reperfusion lung injury after PEA which can be delayed for days before onset [71], the injury associated with BPA appears to be more vascular injury related to the intervention than the capillary leak syndrome described post-PEA [72]. Table 4 is proposed as a guide for BPA centres for classification of complications. Injury caused by wire perforation or interruption of the diseased vessel is the most common [69]. Lung injury by wire perforation or balloon overdilatation in the setting of severe $\mathrm{PH}$ risks potentially fatal massive infiltration and/or haemorrhage which may require mechanical ventilation or extracorporeal support. Classic reperfusion lung injury is rare with BPA. Published low BPA complications reflect limited experience confined to experienced BPA centres. In experienced hands, BPA has emerged as a promising and established treatment for inoperable CTEPH.

\section{PH-targeted medical therapy}

While PEA remains the treatment of choice for most patients with CTEPH, around $40 \%$ of the patients in the international CTEPH registry were considered inoperable due to concern for inaccessible vascular obstruction, PAP out of proportion to morphological lesions and significant prohibitive comorbidities [9]. A large number of small studies and three large randomised controlled trials (table 5) have demonstrated varying improvements with targeted medical therapy in technically inoperable patients [55, 73, 74].

\footnotetext{
TABLE 4 Balloon pulmonary angioplasty complications

During the procedure

Vascular injury" with/without haemoptysis

Wire perforation

Balloon overdilatation

High-pressure contrast injection

Vascular dissection

Allergic reaction to contrast

Adverse reaction to conscious sedation/local anaesthesia

After the procedure

Lung injury" (radiographic opacity with/without haemoptysis, with/without hypoxaemia)

Renal dysfunction

Access site problems

\#: signs of vascular injury: extravasation of contrast, hypoxaemia, cough, tachycardia, increased pulmonary arterial pressure; ${ }^{\text {I: }}$ causes of lung injury: vascular injury much greater than reperfusion lung injury.
} 
TABLE 5 Pulmonary hypertension-targeted medical therapy randomised controlled trials in chronic thromboembolic pulmonary hypertension

\begin{tabular}{|c|c|c|c|c|c|c|c|c|}
\hline Trial [ref.] & Study drug & Duration weeks & Subjects $n$ & NYHA FC & 6MWD m & 6MWD effect $m$ & PVR baseline dyn $\cdot s \cdot \mathrm{cm}^{-5}$ & PVR effect $\%$ \\
\hline CHEST-1 [55] & Riociguat & 16 & 261 & II-IV & $347 \pm 80$ & +46 & $787 \pm 422$ & -31 \\
\hline
\end{tabular}

Data are presented as $\mathrm{n}$ or mean $\pm \mathrm{SD}$, unless otherwise stated. NYHA FC: New York Heart Association Functional Class; 6MWD: 6-min walk distance; PVR: pulmonary vascular resistance; NS: non-significant. All three trials had an adjudication process for operability. ${ }^{\#}$ : 6MWD measured at 24 weeks.

However, data are lacking for patients with medical contraindications or those refusing surgery. Riociguat is the currently approved medical therapy in many countries for inoperable CTEPH based on the CHEST trials $[55,75]$. Recently, the MERIT-1 trial of macitentan in the treatment of inoperable CTEPH showed improvements of the primary end-point (PVR $(\mathrm{p}=0.041)$ ) and of other end-points (e.g. 6-min walk distance $(\mathrm{p}=0.033)$ and $\mathrm{N}$-terminal pro-brain natriuretic peptide $(\mathrm{p}=0.040))$ [74]. This last study provided the first evidence on combination drug therapy in CTEPH. $61 \%$ of the included patients were already treated with phosphodiesterase type 5 inhibitors and/or oral/inhaled prostanoids at inclusion, and addition of macitentan showed similar efficacy compared with the drug-naive patients. Accordingly, macitentan is being considered for potential CTEPH registration. Event-driven morbidity/mortality studies have not been performed in CTEPH.

Patients with persistent/residual post-operative $\mathrm{PH}$ were also included in BENEFIT and CHEST-1, representing around $30 \%$ of the study population $[55,73]$. Both studies included patients with mPAP $\geqslant 25 \mathrm{mmHg}$ and PVR $\geqslant 300 \mathrm{dyn} \cdot \mathrm{s} \cdot \mathrm{cm}^{-5}$ at $>6$ months after endarterectomy. This can be put in perspective with real-life data from the large UK national cohort, in which 3-6 months after PEA: 1) 51\% of the patients had $\mathrm{mPAP} \geqslant 25 \mathrm{mmHg}, 2) \mathrm{mPAP} \geqslant 30 \mathrm{mmHg}$ predicted initiation of $\mathrm{PH}$-targeted medical therapy, and 3) $\mathrm{mPAP} \geqslant 38 \mathrm{mmHg}$ and PVR $\geqslant 425$ dyn $\cdot \mathrm{s} \cdot \mathrm{cm}^{-5}$ correlated with worse long-term survival [49].

Using medical therapy as a "bridge to PEA" is more controversial, and is felt to delay timely surgical referral and, therefore, definitive treatment. In the international registry and in a University of California San Diego cohort, $28 \%$ and up to $37 \%$, respectively, of the patients were on some form of PH-targeted $\operatorname{drug}(\mathrm{s})$ at the time of surgical referral $[9,76]$. In both cohorts, the delay between diagnosis and surgery was doubled in the pre-treated patients, without demonstrable clinical benefit. In the international registry, pre-treatment even independently predicted worse outcome (hazard ratio 2.62; p=0.0072) [48]. Key limitations of these reports are with inherent referral bias and the possibility of medical therapy potentially stabilising otherwise deteriorating cases (unknown and not tested). In order to provide the missing evidence, a phase 2 study will soon commence to include CTEPH patients with high PVR for pre-operative treatment with riociguat versus placebo (ClinicalTrials.gov identifier NCT0327357). Using medical therapy as a "bridge to BPA", although not studied, has become common practice and in keeping with the indication for riociguat for technically inoperable disease. A study is currently ongoing which compares riociguat versus BPA for technically inoperable CTEPH, followed by an opportunity to crossover after 6 months (ClinicalTrials.gov identifier NCT02634203).

\section{CTEPH treatment algorithm}

The newly proposed CTEPH treatment algorithm is provided in figure 1 and starts with lifelong anticoagulation. Antiplatelet therapy is not an alternative to anticoagulation in patients with CTEPH. Data differentiating the best form of anticoagulation therapy is lacking in CTEPH. Traditional anticoagulation has been with oral vitamin $\mathrm{K}$ antagonists. Whether the newer oral anticoagulants or chronic injectable anticoagulants are adequate in CTEPH is unknown. The algorithm emphasises the need for a multidisciplinary assessment, including a surgeon experienced with PEA, PH specialist, BPA interventionist and CTEPH-trained radiologist. A PH referral centre was previously defined and recommended as a minimum volume of 50 pulmonary arterial hypertension or CTEPH patients managed per year [77]. However, given the highly specialised nature of CTEPH treatment, additional factors should be considered when gauging clinical expertise.

In the CHEST-1 trial, central adjudication exemption and local operability assessment were allowed provided a participating centre performed more than 20 PEA operations per year [78]. However, the majority of the operability adjudication occurred with the central committee whose members each 


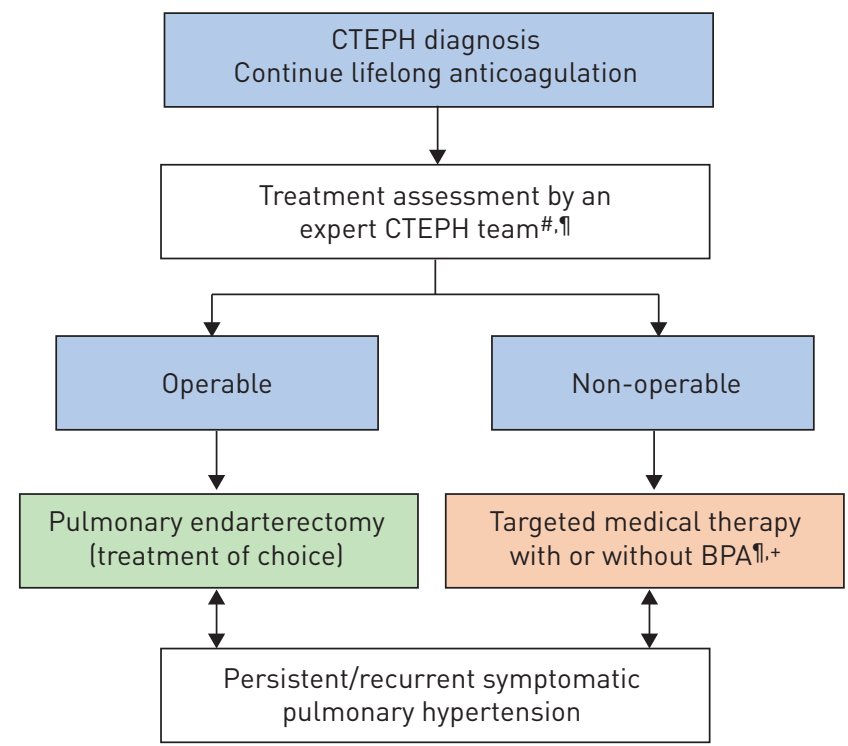

FIGURE 1 Chronic thromboembolic pulmonary hypertension (CTEPH): revised treatment algorithm. BPA: balloon pulmonary angioplasty. \#: multidisciplinary: pulmonary endarterectomy surgeon, PH expert, BPA interventionist and radiologist; ": treatment assessment may differ depending on the level of expertise; ${ }^{+}$: BPA without medical therapy can be considered in selected cases.

performed well in excess of 50 operations per year. In addition, the central adjudication committee had double the rate of operability determination than the local adjudication committee (15\% operable versus 7\% operable, respectively). From the international CTEPH registry, a trend with the best in-hospital and 1-year post-operative mortality was observed from centres performing higher volumes of PEA, with the best results observed from centres performing more than 50 operations per year [79]. This observation likely does not take into account the relative differences in case complexity, with potentially more challenging cases referred to higher-volume programmes. Additional emphasis on the importance of surgical centre experience was reported in the UK national registry of patients undergoing PEA [49]. In this report, significantly lower in-hospital mortality was observed in the second group of consecutive 500 operated cases compared with the initial group of 500 operated cases. In addition, the ability for high-volume centres to perform more distal endarterectomy necessitates stratification of surgical centre expertise [53]. A similar observation applies to BPA success; the safety and efficacy reports of the refined BPA techniques from Japan are from centres performing the highest volume of procedures (typically more than 100 per year) $[58,59]$. In summary, an expert CTEPH centre should be able to assess and deliver all established treatment modalities with outcomes similar to or exceeding those published.

Patients with operable CTEPH should receive PEA as the treatment of choice. For those deemed inoperable, the best level of evidence supports initiating medical therapy and consideration of BPA. Patients with persistent/recurrent symptomatic PH following PEA should receive medical therapy and be considered for BPA or re-do endarterectomy in cases of significant re-occlusion [80]. Lastly, given the subjectivity of operability assessment, it is possible for a patient initially deemed to be inoperable to receive PEA with or without treatments for inoperable CTEPH. The new algorithm therefore allows for fluidity between these treatment modalities as information and expertise is gained.

\section{Conclusions}

PEA remains the treatment of choice for patients with operable CTEPH. Two additional recognised treatments are now available (i.e. targeted medical therapy and BPA). A multimodal, individualised approach to treatment at expert centres integrating surgical, interventional, imaging and medical $\mathrm{PH}$ expertise with the development of clear outcomes analyses is mandatory going forward.

Conflict of interest: N.H. Kim reports personal fees for consultancy, steering committee work and speaker bureau membership from Actelion and Bayer; personal fees for consultancy from Merck; and is a board member of the International CTEPH Association, CTEPH.com. M. Delcroix is an investigator, speaker, consultant or steering committee member for Actelion, Bayer AG, Bellerophon, Eli Lilly, GSK, MSD, Pfizer and Reata; and has received an institutional research grant from Actelion. X. Jais received grants and personal fees from Actelion, GSK, Bayer and MSD. M.M. Madani has received consultancy fees from MSD/Bayer, Wexler Surgical and Actelion, and is an executive board member of the International CTEPH Association, CTEPH.com. H. Matsubara has received lecture fees from 
Actelion Pharmaceuticals Japan, Ltd, AOP orphan Pharmaceuticals AG, Bayer Yakuhin, Ltd, Pfizer Japan, Inc, Nippon Shinyaku, Co, Ltd and Kaneka Medix Corporation, outside the submitted work. E. Mayer has received consultancy fees from Actelion, Bayer and MSD; and is on the speaker bureau for Actelion, Bayer, MSD and Pfizer. T. Ogo is a member of the speaker bureau for Actelion and Bayer. V.F. Tapson received research support from Actelion, Arena, Bayer, $\mathrm{BiO} 2$, Edwards Scientific, Ekos/BTG, Janssen, Inari, Portola, Reata, United Therapeutics, Daiichi and Penumbra; consultancy fees from Actelion, Bayer, BiO2, Ekos/BTG, Inari, Janssen, Portola, United Therapeutics, VWave, Daiichi and Penumbra; speaker bureau for Actelion, Bayer and Janssen. H-A. Ghofrani reports personal fees for advisory board work payment for lectures including service on speaker bureaus from Actelion, Bayer, GSK, Novartis and Pfizer; consultancy fees from Actelion, Bayer, Bellerophon Pulse Technologies, GSK, MSD, Novartis and Pfizer; and grants from Deutsche Forschungsgemeinschaft (DFG), outside the submitted work. D.P. Jenkins worked as adjudicator for Actelion and Bayer for MERIT and CHEST-1 studies; received honoraria for speaking from Actelion and Bayer; and is a board member of the International CTEPH Association, CTEPH.com.

\section{References}

1 Simonneau G, Montani D, Celermajer DS, et al. Haemodynamic definitions and updated clinical classification of pulmonary hypertension. Eur Respir J 2019; 53: 1801913.

2 Lang IM, Pesavento R, Bonderman D, et al. Risk factors and basic mechanisms of chronic thromboembolic pulmonary hypertension: a current understanding. Eur Respir J 2013; 41: 462-468.

3 Moser KM, Bloor CM. Pulmonary vascular lesions occurring in patients with chronic major vessel thromboembolic pulmonary hypertension. Chest 1993; 103: 685-692.

4 Dorfmüller P, Günther S, Ghigna M-R, et al. Microvascular disease in chronic thromboembolic pulmonary hypertension: a role for pulmonary veins and systemic vasculature. Eur Respir J 2014; 44: 1275-1288.

5 Kim NH, Delcroix M, Jenkins DP, et al. Chronic thromboembolic pulmonary hypertension. J Am Coll Cardiol 2013; 62: D92-D99.

6 Fedullo P, Kerr KM, Kim NH, et al. Chronic thromboembolic pulmonary hypertension. Am J Respir Crit Care Med 2011; 183: 1605-1613.

7 Simonneau G, Torbicki A, Dorfmüller P, et al. The pathophysiology of chronic thromboembolic pulmonary hypertension. Eur Respir Rev 2017; 26: 160112.

8 Egermayer P, Peacock AJ. Is pulmonary embolism a common cause of chronic pulmonary hypertension? Limitations of the embolic hypothesis. Eur Respir J 2000; 15: 440-448.

9 Pepke-Zaba J, Delcroix M, Lang I, et al. Chronic thromboembolic pulmonary hypertension (CTEPH): results from an international prospective registry. Circulation 2011; 124: 1973-1981.

10 Ogawa A, Satoh T, Fukuda T, et al. Balloon pulmonary angioplasty for chronic thromboembolic pulmonary hypertension: results of a multicenter registry. Circ Cardiovasc Qual Outcomes 2017; 10: e004029.

11 Tanabe N. Analysis of Chronic Thromboembolic Pulmonary Hypertension (Intractable Disease Database). Tokyo, Ministry of Health, Wealth and Labor, 2008.

$12 \mathrm{Gu}$ S, Su P, Yan J, et al. Comparison of gene expression profiles and related pathways in chronic thromboembolic pulmonary hypertension. Int J Mol Med 2014; 33: 277-300.

13 Pengo V, Lensing AW, Prins MH, et al. Incidence of chronic thromboembolic pulmonary hypertension after pulmonary embolism. N Engl J Med 2004; 350: 2257-2264.

14 Becattini C, Agnelli G, Pesavento R, et al. Incidence of chronic thromboembolic pulmonary hypertension after a first episode of pulmonary embolism. Chest 2006; 130: 172-175.

15 Klok FA, van Kralingen KW, van Dijk AP, et al. Prospective cardiopulmonary screening program to detect chronic thromboembolic pulmonary hypertension in patients after acute pulmonary embolism. Haematologica 2010; 95: 970-975.

16 Martí D, Gómez V, Escobar C, et al. Incidencia de hipertensión pulmonar tromboembólica crónica sintomática y asintomática. [Incidence of symptomatic and asymptomatic chronic thromboembolic pulmonary hypertension.] Arch Bronconeumol 2010; 46: 628-633.

17 Poli D, Grifoni E, Antonucci E, et al. Incidence of recurrent venous thromboembolism and of chronic thromboembolic pulmonary hypertension in patients after a first episode of pulmonary embolism. $J$ Thromb Thrombolysis 2010; 30: 294-299.

18 Surie S, Gibson NS, Gerdes VE, et al. Active search for chronic thromboembolic pulmonary hypertension does not appear indicated after acute pulmonary embolism. Thromb Res 2010; 125: e202-e205.

19 Berghaus TM, Barac M, von Scheidt W, et al. Echocardiographic evaluation for pulmonary hypertension after recurrent pulmonary embolism. Thromb Res 2011; 128: e144-e147.

20 Held M, Hesse A, Gött F, et al. A symptom-related monitoring program following pulmonary embolism for the early detection of CTEPH: a prospective observational registry study. BMC Pulm Med 2014; 14: 141.

21 Giuliani L, Piccinino C, D'Armini MA, et al. Prevalence of undiagnosed chronic thromboembolic pulmonary hypertension after pulmonary embolism. Blood Coagul Fibrinolysis 2014; 25: 649-653.

22 Guérin L, Couturaud F, Parent F, et al. Prevalence of chronic thromboembolic pulmonary hypertension after acute pulmonary embolism. Thromb Haemost 2014; 112: 598-605.

23 Kayaalp I, Varol Y, Cimen P, et al. The incidence of chronic thromboembolic pulmonary hypertension secondary to acute pulmonary thromboembolism. Tuberk Toraks 2014; 62: 199-206.

24 Vavera Z, Vojacek J, Pudil R, et al. Chronic thromboembolic pulmonary hypertension after the first episode of pulmonary embolism? How often? Biomed Pap Med Fac Univ Palacky Olomouc Czech Repub 2016; 160: 125-129.

25 Klok FA, Tesche C, Rappold L, et al. External validation of a simple non-invasive algorithm to rule out chronic thromboembolic pulmonary hypertension after acute pulmonary embolism. Thromb Res 2015; 135: 796-801.

26 Coquoz N, Weilenmann D, Stolz D, et al. Multicentre observational screening survey for the detection of CTEPH following pulmonary embolism. Eur Respir J 2018; 51: 1702505.

27 Lang IM. Chronic thromboembolic pulmonary hypertension - not so rare after all. N Engl J Med 2004; 350 2236-2238.

28 Tapson VF, Humbert M. Incidence and prevalence of chronic thromboembolic pulmonary hypertension: from acute to chronic pulmonary embolism. Proc Am Thorac Soc 2006; 3: 564-567. 
29 McLaughlin VV, Langer A, Tan M, et al. Contemporary trends in the diagnosis and management of pulmonary arterial hypertension an initiative to close the care gap. Chest 2013; 143: 324-332.

30 Tapson VF, Platt DM, Xia F, et al. Monitoring for pulmonary hypertension following pulmonary embolism: the INFORM study. Am J Med 2016; 129: 978-985.

31 Oger E. Incidence of venous thromboembolism: a community-based study in Western France. Thromb Haemost 2000; 83: 657-660.

32 Sharifi M, Bay C, Skrocki L, et al. Moderate pulmonary embolism treated with thrombolysis (from the MOPETT Trial). Am J Cardiol 2013; 111: 273-277.

33 Konstantinides SV, Vicaut E, Danays T, et al. Impact of thrombolytic therapy on the long-term outcome of intermediate-risk pulmonary embolism. J Am Coll Cardiol 2017; 69: 1536-1544.

34 van Kan $\mathrm{C}$, van der Plas MN, Reesink $\mathrm{HJ}$, et al. Hemodynamic and ventilatory responses during exercise in chronic thromboembolic disease. J Thorac Cardiovasc Surg 2016; 152: 763-771.

35 Held M, Kolb P, Grün M, et al. Functional characterization of patients with chronic thromboembolic disease. Respiration 2016; 91: 503-509.

36 Klok FA, Van Kralingen KW, Van Dijk APJ, et al. Prevalence and potential determinants of exertional dyspnea after acute pulmonary embolism. Respir Med 2010; 104: 1744-1749.

37 Nijkeuter M, Hovens MMC, Davidson BL, et al. Resolution of thromboemboli in patients with acute pulmonary embolism: a systematic review. Chest 2006; 129: 192-197.

38 Taboada D, Pepke-Zaba J, Jenkins DP, et al. Outcome of pulmonary endarterectomy in symptomatic chronic thromboembolic disease. Eur Respir J 2014; 44: 1635-1645.

39 Tunariu N, Gibbs SJ, Win Z, et al. Ventilation-perfusion scintigraphy is more sensitive than multidetector CTPA in detecting chronic thromboembolic pulmonary disease as a treatable cause of pulmonary hypertension. $J$ Nucl Med 2007; 48: 680-684.

40 He J, Fang W, Lv B, et al. Diagnosis of chronic thromboembolic pulmonary hypertension: comparison of ventilation/perfusion scanning and multidetector computed tomography pulmonary angiography with pulmonary angiography. Nucl Med Commun 2012; 33: 459-463.

41 Rajaram S, Swift AJ, Telfer A, et al. 3D contrast-enhanced lung perfusion MRI is an effective screening tool for chronic thromboembolic pulmonary hypertension: results from the ASPIRE Registry. Thorax 2013; 68: $677-678$.

42 Johns CS, Swift AJ, Rajaram S, et al. Lung perfusion: MRI vs. SPECT for screening in suspected chronic thromboembolic pulmonary hypertension. J Magn Reson Imaging 2017; 46: 1693-1697.

43 Ley S, Ley-Zaporozhan J, Pitton MB, et al. Diagnostic performance of state-of-the-art imaging techniques for morphological assessment of vascular abnormalities in patients with chronic thromboembolic pulmonary hypertension (CTEPH). Eur Radiol 2012; 22: 607-616.

44 Reichelt A, Hoeper MM, Galanski M, et al. Chronic thromboembolic pulmonary hypertension: evaluation with 64-detector row CT versus digital substraction angiography. Eur J Radiol 2009; 71: 49-54.

45 Sugiura T, Tanabe N, Matsuura Y, et al. Role of 320-slice CT imaging in the diagnostic workup of patients with chronic thromboembolic pulmonary hypertension. Chest 2013; 143: 1070-1077.

46 Shimizu H, Tanabe N, Terada J, et al. Dilatation of bronchial arteries correlates with extent of central disease in patients with chronic thromboembolic pulmonary hypertension. Circ J 2008; 72: 1136-1141.

47 Ogo T, Fukuda T, Tsuji A, et al. Efficacy and safety of balloon pulmonary angioplasty for chronic thromboembolic pulmonary hypertension guided by cone-beam computed tomography and electrocardiogram-gated area detector computed tomography. Eur J Radiol 2017; 89: 270-276.

48 Delcroix M, Lang I, Pepke-Zaba J, et al. Long-term outcome of patients with chronic thromboembolic pulmonary hypertension: results from an international prospective registry. Circulation 2016; 133: 859-871.

49 Cannon JE, Su L, Kiely DG, et al. Dynamic risk stratification of patient long-term outcome after pulmonary endarterectomy: results from the united kingdom national cohort. Circulation 2016; 133: 1761-1771.

50 Madani M, Mayer E, Fadel E, et al. Pulmonary endarterectomy. Patient selection, technical challenges, and outcomes. Ann Am Thorac Soc 2016; 13: Suppl. 3, S240-S247.

51 D’Armini AM, Morsolini M, Mttiucci G, et al. Pulmonary endarterectomy for distal chronic thromboembolic pulmonary hypertension. J Thorac Cardiovasc Surg 2014; 148: 1005-1011.

52 Thistlethwaite PA, Mo M, Madani MM, et al. Operative classification of thromboembolic disease determines outcome after pulmonary endarterectomy. J Thorac Cardiovasc Surg 2002; 124: 1203-1211.

53 Jenkins D, Madani M, Fadel E, et al. Pulmonary endarterectomy in the management of chronic thromboembolic pulmonary hypertension. Eur Respir Rev 2017; 26: 160111.

54 Wiedenroth CB, Liebetrau C, Breithecker A, et al. Combined pulmonary endarterectomy and balloon pulmonary angioplasty in patients with chronic thromboembolic pulmonary hypertension. J Heart Lung Transplant 2016; 35: 591-596.

55 Ghofrani HA, D'Armini AM, Grimminger F, et al. Riociguat for the treatment of chronic thromboembolic pulmonary hypertension. N Engl J Med 2013; 369: 319-329.

56 Mizoguchi H, Ogawa A, Munemasa M, et al. Refined balloon pulmonary angioplasty for inoperable patients with chronic thromboembolic pulmonary hypertension. Circ Cardiovasc Interv 2012; 5: 748-755.

57 Kataoka M, Inami T, Hayashida K, et al. Percutaneous transluminal pulmonary angioplasty for the treatment of chronic thromboembolic pulmonary hypertension. Circ Cardiovasc Interv 2012; 5: 756-762.

58 Sugimura K, Fukumoto Y, Satoh K, et al. Percutaneous transluminal pulmonary angioplasty markedly improves pulmonary hemodynamics and long-term prognosis in patients with chronic thromboembolic pulmonary hypertension. Circ J 2012; 76: 485-488.

59 Ogo T. Balloon pulmonary angioplasty for inoperable chronic thromboembolic pulmonary hypertension. Curr Opin Pulm Med 2015; 21: 425-431.

60 Fukui S, Ogo T, Goto $\mathrm{Y}$, et al. Exercise intolerance and ventilatory inefficiency improve early after balloon pulmonary angioplasty in patients with inoperable chronic thromboembolic pulmonary hypertension. Int $J$ Cardiol 2015; 180: 66-68.

61 Fukui S, Ogo T, Morita Y, et al. Right ventricular reverse remodelling after balloon pulmonary angioplasty. Eur Respir J 2014; 43: 1394-1402. 
62 Feinstein JA, Goldhaber SZ, Lock JE, et al. Balloon pulmonary angioplasty for treatment of chronic thromboembolic pulmonary hypertension. Circulation 2001; 103: 10-13.

63 Inami $\mathrm{T}$, Kataoka $\mathrm{M}$, Yanagisawa $\mathrm{R}$, et al. Long-term outcomes after percutaneous transluminal pulmonary angioplasty for chronic thromboembolic pulmonary hypertension. Circulation 2016; 134: 2030-2032.

64 Andreassen AK, Ragnarsson A, Gude E, et al. Balloon pulmonary angioplasty in patients with inoperable chronic thromboembolic pulmonary hypertension. Heart 2013; 99: 1415-1420.

65 Bouvaist $\mathrm{H}$, Thony $\mathrm{F}$, Jondot $\mathrm{M}$, et al. Balloon pulmonary angioplasty in a patient with chronic thromboembolic pulmonary hypertension. Eur Respir Rev 2014; 23: 393-395.

66 Roik M, Wretowski D, Rowinski O, et al. Refined balloon pulmonary angioplasty in inoperable chronic thromboembolic pulmonary hypertension - a multi-modality approach to the treated lesion. Int J Cardiol 2014; 177: e139-e141.

67 Olsson KM, Wiedenroth CB, Kamp J-C, et al. Balloon pulmonary angioplasty for inoperable patients with chronic thromboembolic pulmonary hypertension: the initial German experience. Eur Respir J 2017; 49: 1602409.

68 Ogawa A, Matsubara H. Balloon pulmonary angioplasty: a treatment option for inoperable patients with chronic thromboembolic pulmonary hypertension. Front Cardiovasc Med 2015; 2: 4.

69 Kawakami T, Ogawa A, Miyaji K, et al. Novel angiographic classification of each vascular lesion in chronic thromboembolic pulmonary hypertension based on selective angiogram and results of balloon pulmonary angioplasty. Circ Cardiovasc Interv 2016; 9: e003318.

70 Roik M, Wretowski D, Labyk A, et al. Refined balloon pulmonary angioplasty driven by combined assessment of intra-arterial anatomy and physiology - multimodal approach to treated lesions in patients with non-operable distal chronic thromboembolic pulmonary hypertension - technique, safety and efficacy of 50 consecutive angioplasties. Int J Cardiol 2016; 15: 228-235.

71 Kerr KM, Auger WR, Marsh JJ, et al. The use of cylexin (CY-1503) in prevention of reperfusion lung injury in patients undergoing pulmonary thromboendarterectomy. Am J Respir Crit Care Med 2000; 162: 14-20.

72 Lang I, Meyer BC, Ogo T, et al. Balloon pulmonary angioplasty in chronic thromboembolic pulmonary hypertension. Eur Respir Rev 2017; 26: 160119.

73 Jais X, D'Armini AM, Jansa P, et al. Bosentan for treatment of inoperable chronic thromboembolic pulmonary hypertension: BENEFiT (Bosentan Effects in iNopErable Forms of chronIc Thromboembolic pulmonary hypertension), a randomized, placebo-controlled trial. J Am Coll Cardiol 2008; 52: 2127-2134.

74 Ghofrani HA, Simonneau G, D’Armini AM, et al. Macitentan for the treatment of inoperable chronic thromboembolic pulmonary hypertension (MERIT-1): results from the multicentre, phase 2, randomised, double-blind, placebo-controlled study. Lancet Respir Med 2017; 5: 785-794.

75 Simonneau G, D'Armini AM, Ghofrani HA, et al. Predictors of long-term outcomes in patients treated with riociguat for chronic thromboembolic pulmonary hypertension: data from the CHEST-2 open-label, randomised, long-term extension trial. Lancet Respir Med 2016; 4: 372-380.

76 Jensen KW, Kerr KM, Fedullo PF, et al. Pulmonary hypertensive medical therapy in chronic thromboembolic pulmonary hypertension before pulmonary thromboendarterectomy. Circulation 2009; 120: 1248-1254.

77 Galiè N, Humbert M, Vachiery JL, et al. 2015 ESC/ERS Guidelines for the diagnosis and treatment of pulmonary hypertension. Eur Respir J 2015; 46: 903-975.

78 Jenkins DP, Biederman A, D’Armini AM, et al. Operability assessment in CTEPH: lessons from the CHEST-1 study. J Thorac Cardiovasc Surg 2016; 152: 669-674.

79 Mayer E, Jenkins D, Lindner J, et al. Surgical management and outcome of patients with chronic thromboembolic pulmonary hypertension: results from an international prospective registry. J Thorac Cardiovasc Surg 2011; 141: $702-710$.

80 Mo M, Kapelanski DP, Mitruka SN, et al. Reoperative pulmonary thromboendarterectomy. Ann Thorac Surg 1999; 68: $1770-1777$. 